OPEN ACCESS

Edited by:

Bryce Chackerian,

University of New Mexico,

United States

Reviewed by:

Paolo Martelletti,

Sapienza University of Rome, Italy

Hsiangkuo Yuan,

Thomas Jefferson University,

United States

*Correspondence:

Roger Cady

ROCD@/undbeck.com

${ }^{\dagger}$ Currently an independent consultant, Saratoga Springs, NY, United States

${ }^{\text {} C}$ Currently with Global Safety Docs, LLC, Paradise Valley, AZ,

United States

$\S_{\text {No current affiliation }}$

Specialty section:

This article was submitted to Vaccines and Molecular Therapeutics,

a section of the journal

Frontiers in Immunology

Received: 27 August 2021 Accepted: 05 October 2021 Published: 25 October 2021

Citation:

Pederson S, Biondi DM, Allan B, Cady R, Schaeffler B, Baker B and Latham J (2021) Clinical Immunogenicity Evaluation of

Eptinezumab, a Therapeutic Humanized Monoclonal Antibody Targeting Calcitonin Gene-Related Peptide (CGRP) for the Preventive

Treatment of Migraine.

Front. Immunol. 12:765822. doi: 10.3389/fimmu.2021.765822

\section{Clinical Immunogenicity Evaluation of Eptinezumab, a Therapeutic Humanized Monoclonal Antibody Targeting Calcitonin Gene-Related Peptide (CGRP) for the Preventive Treatment of Migraine}

\author{
Susan Pederson ${ }^{1}$, David M. Biondi ${ }^{2 \dagger}$, Brent Allan ${ }^{2 \neq}$, Roger Cady ${ }^{1 *}$, Barbara Schaeffler ${ }^{1}$, \\ Brian Baker ${ }^{1}$ and John Latham ${ }^{2 \S}$ \\ ${ }^{1}$ Lundbeck Seattle BioPharmaceuticals, Inc., Bothell, WA, United States, ${ }^{2}$ Alder BioPharmaceuticals, Inc. (CKA Lundbeck \\ Seattle BioPharmaceuticals, Inc.), Bothell, WA, United States
}

Background: Eptinezumab is a humanized monoclonal antibody that selectively binds calcitonin gene-related peptide and is indicated for the preventive treatment of migraine in adults. This analysis characterizes the immunogenic profile of eptinezumab using data from clinical trials of eptinezumab for migraine prevention.

Methods: Immunogenicity data were collected from five studies that included 2076 patients with episodic or chronic migraine treated with eptinezumab at dose levels ranging from 10 to 1000 mg, administered intravenously for up to 4 doses at 12-week intervals. Anti-drug antibody (ADA) results were available from 2074 of these patients. Four studies were randomized, double-blind, placebo-controlled trials with ADA monitoring for up to 56 weeks; one was a 2-year, open-label, phase 3 safety study with ADA monitoring for 104 weeks. Patients who had a confirmed ADA-positive result at the end-of-study visit were monitored for up to 6 additional months. Development of ADA and neutralizing antibodies (NAbs) were evaluated to explore three key areas of potential impact: pharmacokinetic exposure profile (eptinezumab trough plasma concentrations), efficacy (change in monthly migraine days), and safety (rates of treatment-emergent adverse events). These studies included methods designed to capture the dynamics of a potential humoral immune response to eptinezumab treatment, and descriptive analyses were applied to interpret the relationship of ADA signals to drug exposure, efficacy, and safety.

Results: Pooled across the five clinical trials, treatment-emergent ADAs and NAbs occurred in 15.8 and $6.2 \%$ of eptinezumab-treated patients, respectively. Highly consistent profiles were observed across all studies, with initial onset of detectable ADA observed at the week 8 measurement and maximal ADA frequency and titer observed at 
week 24, regardless of eptinezumab dose level or number of doses. After 24 weeks, the ADA and NAb titers steadily declined despite additional doses of eptinezumab.

Interpretation: Collectively, these integrated analyses did not demonstrate any clinically meaningful impact from ADA occurring after treatment with eptinezumab. The ADA profiles were low titer and transient, with the incidence and magnitude of ADA or NAb responses declining after week 24 . Development of ADAs and NAbs did not impact the efficacy and safety profiles of eptinezumab.

Keywords: eptinezumab, immunogenicity, anti-drug antibody, monoclonal antibody, migraine, neutralizing antibody, calcitonin gene-related peptide (CGRP)

\section{INTRODUCTION}

Eptinezumab, a calcitonin gene-related peptide (CGRP) antagonist indicated for the preventive treatment of migraine in adults (1), is a humanized immunoglobulin G1 (IgG1) monoclonal antibody $(\mathrm{mAb})$ that binds CGRP with high affinity (2). It has demonstrated migraine preventive effects across the spectrum of migraine attack frequency, with statistically significant reduction from baseline in migraine frequency established as early as 1 day after the initial infusion and sustained throughout the 12-week dosing interval, with an acceptable safety and tolerability profile (3-11).

Like almost all biological proteins, $\mathrm{mAbs}$ have the potential to trigger an immune response after administration (12-16). In most cases, associated immunogenicity is multifactorial, with intrinsic factors, systems biology, conditions of use, patientrelated factors, and product quality all potentially contributing. As with other humanized and fully recombinant human $\operatorname{IgG} 1$ mAbs (13), the non-human germline sequences in the complementarity-determining regions (CDRs) are the structural features of eptinezumab with the greatest immunogenic potential. Eptinezumab contains a human IgG1 Fc region sequence that corresponds to the two most common allotypes, G1m1,17 and G1m3, with the single exception of position 297, which was changed from an asparagine to an alanine to prevent $\mathrm{N}$-linked glycosylation of the heavy chain. The CDR sequences are derived from a parental rabbit anti-alpha CGRP antibody; the six CDR sequences from the original rabbit antibody $\mathrm{VL}$ and $\mathrm{VH}$ regions were grafted into human VL and VH framework sequences that were most similar to the rabbit framework sequence. To retain full affinity of the humanized antibody for CGRP, several amino acids in the human framework were substituted with the corresponding rabbit amino acid to preserve antigen-binding affinity. The remainder of the variable regions represent a human germline sequence.

The clinical development of eptinezumab included assessments of immunogenicity. Comprehensive evaluations were performed to detect and characterize immune responses observed within the studies and to identify any correlation of immunogenicity with pharmacokinetic (PK), pharmacodynamic, efficacy, and safety endpoints. The objective of the integrated summary of immunogenicity analysis was to pool immunogenicity data from the five clinical studies of eptinezumab conducted in patients with migraine in order to robustly characterize the immunogenic profile of eptinezumab within this population.

\section{MATERIALS AND METHODS}

\section{Study Designs}

The clinical development program of eptinezumab in migraine prevention included four randomized, double-blind, placebocontrolled trials and one open-label safety study (Table 1). Approval for each study was provided by the independent ethics committee or institutional review board of the study sites. All studies were conducted in accordance with Good Clinical Practice guidelines, the principles of the Declaration of Helsinki, and all applicable regulatory requirements. Patients provided written informed consent prior to initiation of any study procedures. Each study is registered on ClinicalTrials.gov (NCT01772524, NCT02275117, NCT02559895, NCT02974153, NCT02985398).

Two of the placebo-controlled trials were single-dose studies: one in patients with episodic migraine [study 1 (3)] and one in patients with chronic migraine [study 2 (4)]. The remaining three trials were multiple-dose studies: PROMISE-1 evaluated eptinezumab for up to 4 doses ( 1 year) in patients with episodic migraine $(5,17)$, PROMISE-2 evaluated eptinezumab for up to 2 doses (6 months) in patients with chronic migraine $(7,8)$, and PREVAIL evaluated eptinezumab for up to 8 doses ( 2 years) in patients with chronic migraine (9). Though PREVAIL included 8 doses, the integrated analyses herein only include the interim study data (i.e., the primary treatment phase, or first 4 doses), as the study was still ongoing when the integrated summary of immunogenicity report was finalized for submission of the biological licensing application. In all trials, study drug was administered by intravenous infusion lasting 30 minutes (PROMISE-2 and PREVAIL) or 1 hour (study 1, study 2, and PROMISE-1).

\section{Assessment of Anti-Drug Antibodies}

Immunogenicity sampling time points are shown in Supplemental Table 1. In each study, samples were collected prior to study drug administration on day 0 and regularly throughout each study at similar time points for analysis. Three of the studies included a 2-week time point to evaluate 
TABLE 1 | Overview of clinical studies contributing to the immunogenicity evaluation for eptinezumab.

\begin{tabular}{|c|c|c|c|c|}
\hline Study & $\begin{array}{c}\text { Description } \\
\text { (ClinicalTrials.gov ID) }\end{array}$ & Migraine diagnosis & $\begin{array}{c}\text { Dose levels } \\
\text { Number of doses (schedule) }\end{array}$ & Epti-treated patients with ADA results \\
\hline Study 1 & $\begin{array}{l}\text { Phase 1b, DB/R/PC } \\
\text { (NCT01772524) (3) }\end{array}$ & EM & $\begin{array}{l}1000 \text { mg, placebo } \\
\text { Single dose (day 0) }\end{array}$ & 1000 mg: 81 \\
\hline Study 2 & $\begin{array}{l}\text { Phase 2, DB/R/PC } \\
(\text { NCT02275117) (4) }\end{array}$ & $\mathrm{CM}$ & $\begin{array}{l}\text { 10, 30, 100, } 300 \text { mg, placebo } \\
\text { Single dose (day 0) }\end{array}$ & $\begin{array}{l}300 \text { mg: } 120 \\
100 \text { mg: } 122 \\
30 \text { mg: } 122 \\
10 \text { mg: } 129\end{array}$ \\
\hline PROMISE-1 & $\begin{array}{l}\text { Phase 3, DB/R/PC } \\
\text { (NCT02559895) }(5,17)\end{array}$ & EM & $\begin{array}{l}\text { 30, 100, } 300 \text { mg, placebo } \\
\text { Four doses (day 0, weeks 12, 24, 36) }\end{array}$ & $\begin{array}{l}300 \mathrm{mg}: 224 \\
100 \mathrm{mg}: 223 \\
30 \mathrm{mg}: 219\end{array}$ \\
\hline PROMISE-2 & $\begin{array}{l}\text { Phase 3, DB/R/PC } \\
\text { (NCT02974153) }(7,8)\end{array}$ & $\mathrm{CM}$ & $\begin{array}{l}\text { 100, } 300 \text { mg, placebo } \\
\text { Two doses (day 0, week 12) }\end{array}$ & $\begin{array}{l}300 \mathrm{mg}: 350 \\
100 \mathrm{mg}: 356\end{array}$ \\
\hline PREVAIL & $\begin{array}{l}\text { Open-label } \\
\text { (NCT02985398) (9) }\end{array}$ & $\mathrm{CM}$ & $\begin{array}{l}300 \mathrm{mg} \\
\text { Eight doses }{ }^{\star} \text { (day } 0 \text {, weeks 12, 24, 36, 48, 60, 72, 84) } \\
\text { Total }\end{array}$ & $\begin{array}{l}300 \mathrm{mg}: 128 \\
2074\end{array}$ \\
\hline
\end{tabular}

*In analyses included in this paper, data from only the first 4 doses of PREVAIL are included due to the fact that PREVAIL was ongoing and the interim analysis of the primary treatment phase (first 4 doses) was planned for inclusion in these analyses. ADA, anti-drug antibody; CM, chronic migraine; DB/R/PC, double-blind, randomized, placebo-controlled; EM, episodic migraine; Epti, eptinezumab.

early seroconversion, followed by sampling at 4-week intervals to the end of study (EOS) and with all studies accounting for the half-life of eptinezumab [27 days (18)] by collecting samples at least 20 weeks (5 half-lives) after the last administration. The scheduled duration of anti-drug antibody (ADA) monitoring for the placebo-controlled studies and primary treatment phase of PREVAIL extended to 56 weeks, and for the PREVAIL secondary treatment phase extended to 104 weeks. Patients who tested positive for ADAs at the time of the last study visit were asked to provide up to two additional blood samples for immunogenicity testing at 3-month intervals to evaluate transient vs persistent ADA responses.

Immunogenicity was assessed in serum from all patients who received eptinezumab, using first- and second-generation binding antibody assays that were validated according to industry and regulatory recommendations $(15,16,19-22)$. The first-generation assay supported study 1 ; the second-generation assay supported the remaining four studies and is summarized here. Validation and in-study performance data demonstrated the methods were suitable for their intended use and provided a scientifically sound framework for the immunogenicity assessment of eptinezumab.

The second-generation ADA assay was formatted as a homogeneous electrochemiluminescence (ECL) bridging assay. A sample pre-treatment step was included to improve sensitivity in the presence of residual drug and resulted in a drug-tolerant method for detection of eptinezumab-reactive ADAs of IgG, $\operatorname{IgM}$, and $\operatorname{IgA}$ isotypes. Test samples were diluted 1:50 and, based on a surrogate positive control, the relative sensitivity was determined to be $87.34 \mathrm{ng} / \mathrm{mL}$ and $67.99 \mathrm{ng} / \mathrm{mL}$ for the screening and confirmatory steps of the method, respectively. Inter-assay precision showed a percent coefficient of variability (\%CV) of $9.50 \%$, with drug tolerances of $>1000 \mu \mathrm{g} / \mathrm{mL},>100 \mu \mathrm{g} /$ $\mathrm{mL}, 25 \mu \mathrm{g} / \mathrm{mL}$, and $25 \mu \mathrm{g} / \mathrm{mL}$ for positive controls in the highcontrol set at $2500 \mathrm{ng} / \mathrm{mL}$, mid-control set at $250 \mathrm{ng} / \mathrm{mL}$, screening low-control set at $110 \mathrm{ng} / \mathrm{mL}$, and confirmatory lowcontrol set at $85 \mathrm{ng} / \mathrm{mL}$, respectively.
Sample analysis was performed using a tiered approach that consisted of a screening tier to detect ADAs, a confirmatory tier to ensure detected ADAs were specific to the drug, and a third tier to serve as a semi-quantitative measurement of the amount of antibody (titer) that was present. A fourth tier was also included as a specificity assessment to characterize the epitope binding of the response to distinguish ADAs that were reactive to the antibody framework from ADAs that were reactive with the CDRs.

To complete the immunogenicity testing paradigm, a neutralizing antibody (NAb) assay was developed to characterize confirmed ADAs for neutralizing potential. The $\mathrm{NAb}$ assay was a qualitative ligand-binding assay, formatted as competitive inhibition method, that evaluated the ability of antieptinezumab antibodies to block binding of the drug to the target ligand, human $\alpha$-CGRP. The dynamics of the humoral immune response to eptinezumab using this tiered approach were described in terms of $\mathrm{ADA}$ positive vs negative status, $\mathrm{ADA}$ titer, and NAb positive vs negative status.

\section{Assessment of Pharmacokinetic Exposure, Efficacy, and Safety}

Blood plasma samples for pharmacokinetic evaluation in eptinezumab-treated patients were collected at the same time points outlined in Supplemental Table 1. Trough plasma concentration $\left(\mathrm{C}_{\text {trough }}\right)$ values were based on the sample taken just prior to the next dose administration for treatment visits and at each scheduled visit when available for the PK population. The plasma concentration values used represent all analytically valid plasma concentration results reported.

In all studies, patients recorded migraine and headache data daily using an eDiary. The efficacy outcome used for this analysis was defined as the change from baseline in monthly migraine days (MMDs).

Adverse events were collected from the time of informed consent through the final patient visit. Verbatim descriptions of adverse events were mapped to the Medical Dictionary for 
Regulatory Activities (MedDRA) thesaurus terms and converted to the same version (version 20.1) for all studies. A treatmentemergent adverse event (TEAE) was defined as an adverse event with a start date and time on or after the date and time of first dose of study drug administration. For the current analysis, impact on the incidence of TEAEs and adverse events of special interest (AESIs) was evaluated. AESIs are listed in Supplemental Table 2 .

\section{Statistical Analysis}

Analysis populations included the safety population (all patients who received eptinezumab; summarized by the treatment they received), the full analysis population (all patients who received treatment; summarized by the treatment to which they were randomized), and the $\mathrm{PK}$ population (all patients in the safety population who also had the $\mathrm{PK}$ parameters required for the analysis).

For this analysis, summary statistics, including the number of patients (n), mean, standard deviation, median, minimum, and maximum were evaluated for continuous variables. Generally, the minimum and maximum values were presented to the same decimal precision as the raw values, the mean and median values to one more, and the standard deviation to two more decimal places than the raw values. For categorical variables, per category, the absolute counts (n) and percentages (\%) of patients with data and, if appropriate, the number of patients with missing data, were presented. Percentages are presented to one decimal place.

Details of the missing data rules used for the individual studies are used for these analyses with the exception of the migraine data for study 1 , which was re-calculated to match the other studies. A brief summary of these rules is provided here with additional details reported in the respective study publications. For TEAE, PK, $\mathrm{ADA}$, and NAb data, no missing data imputation was used. For the analysis of MMDs, missing data were imputed using either normalization or a weighted estimate. If migraine day data were missing for $\leq 7$ days in a 28-day interval, the results were normalized to 28 days by multiplying the observed results by the inverse of the completion rate. If migraine day data were missing for $>7$ days in a 28-day interval, a weighted estimate based upon the results of the current and previous months was used, with greater weight being applied to the current month as the completion rate increased. For the pivotal phase 3 studies (PROMISE-1 and PROMISE-2) MMD change from baseline, analysis of covariance (ANCOVA) was performed to estimate the mean, the mean difference from ADA-negative, 95\% confidence interval (95\%CI), and p-value for the ADA-positive subpopulation.

ADA incidence data from study 1 were excluded from the pooled analysis due to the different assessment methodology (first-generation vs second-generation assay) used. The antieptinezumab antibody titer values were not pooled across the studies to evaluate titer by indication; results were summarized separately for each study.

\section{RESULTS}

\section{ADA/NAb Response Dynamics}

The incidence of treatment-emergent ADA across study 2, PROMISE-1, PROMISE-2, and PREVAIL was 15.9\% (316/ $1993)$, with $6.2 \%(124 / 1993)$ of subjects with ADA results exhibiting antibodies with neutralizing potential (Table 2). The time course of $\mathrm{ADA} / \mathrm{NAb}$ development was highly consistent across all four clinical studies (Figure 1), with initial onset of positivity observed at the week 8 measurement and maximal ADA-positive frequency at week 24, regardless of eptinezumab dose level or number of doses. A total of 11 (13.6\%) patients who received a single $1000-\mathrm{mg}$ dose in study 1 had treatmentemergent anti-eptinezumab immunoreactivity results (firstgeneration assay); 4 (4.9\%) had confirmed NAb.

The time-course of evolution of ADA titer by treatment group across study 2, PROMISE-1, PROMISE-2, and PREVAIL are illustrated in Figure 2. In all studies, mean ADA titer was maximal at the week 24 measurement, declining thereafter; there was no clear dose-response relationship between the distribution of ADA titer values and the eptinezumab dose administered.

The fourth tier of the ADA assay, the specificity tier, represents a competitive inhibition format of the ADA screening assay, using ALD306, a Pichia-expressed monoclonal antibody that has the same framework and similar glycosylation profile as ALD403 (eptinezumab), but different CDRs, and CHO-derived ALD403, an antibody molecule produced in $\mathrm{CHO}$ cells with the same CDRs as Pichia-derived ALD403 (eptinezumab). Confirmed ADA positive samples were characterized for specificity. The aggregated results for all sample timepoints were summarized for each study where the signals were mainly reactive with the $\mathrm{CHO}$-expressed eptinezumab, demonstrating specificity for the CDRs of eptinezumab rather than the IgG1 framework region.

TABLE 2 | Incidence of ADA/NAb by study (safety population)*.

\begin{tabular}{|c|c|c|c|c|c|c|}
\hline & \multicolumn{2}{|c|}{ Phase 2} & \multicolumn{3}{|c|}{ Phase 3} & \multirow[t]{2}{*}{ All studies } \\
\hline & Study $1(3)^{*}$ & Study 2 (4) & PROMISE-1 $(5,17)$ & PROMISE-2 $(7,8)$ & PREVAIL (9) & \\
\hline Eptinezumab-treated patients with ADA results, $\mathrm{N}$ & 81 & 493 & 666 & 706 & 128 & 2074 \\
\hline ADA positive at any time point, $\mathrm{n}(\%)^{\dagger}$ & $11(13.6)$ & $59(12.0)$ & $119(17.9)$ & $129(18.3)$ & $22(17.2)$ & $340(16.4)$ \\
\hline Treatment-emergent ADA, n (\%) & $11(13.6)$ & $55(11.2)$ & $116(17.4)$ & $123(17.4)$ & $22(17.2)$ & $327(15.8)$ \\
\hline NAb positive at any time point, $\mathrm{n}(\%)^{\ddagger}$ & $4(4.9)$ & $18(3.7)$ & $52(7.8)$ & $45(6.4)$ & $9(7.0)$ & $128(6.2)$ \\
\hline
\end{tabular}

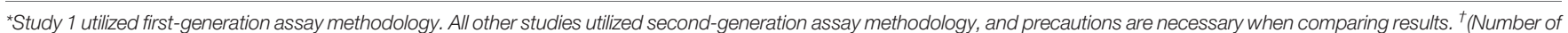

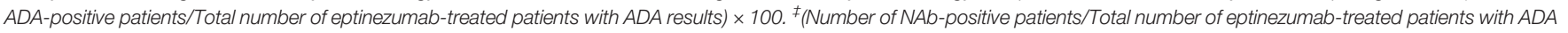
results) $\times 100 . A D A$, anti-drug antibody; $N A b$, neutralizing antibody. 
A
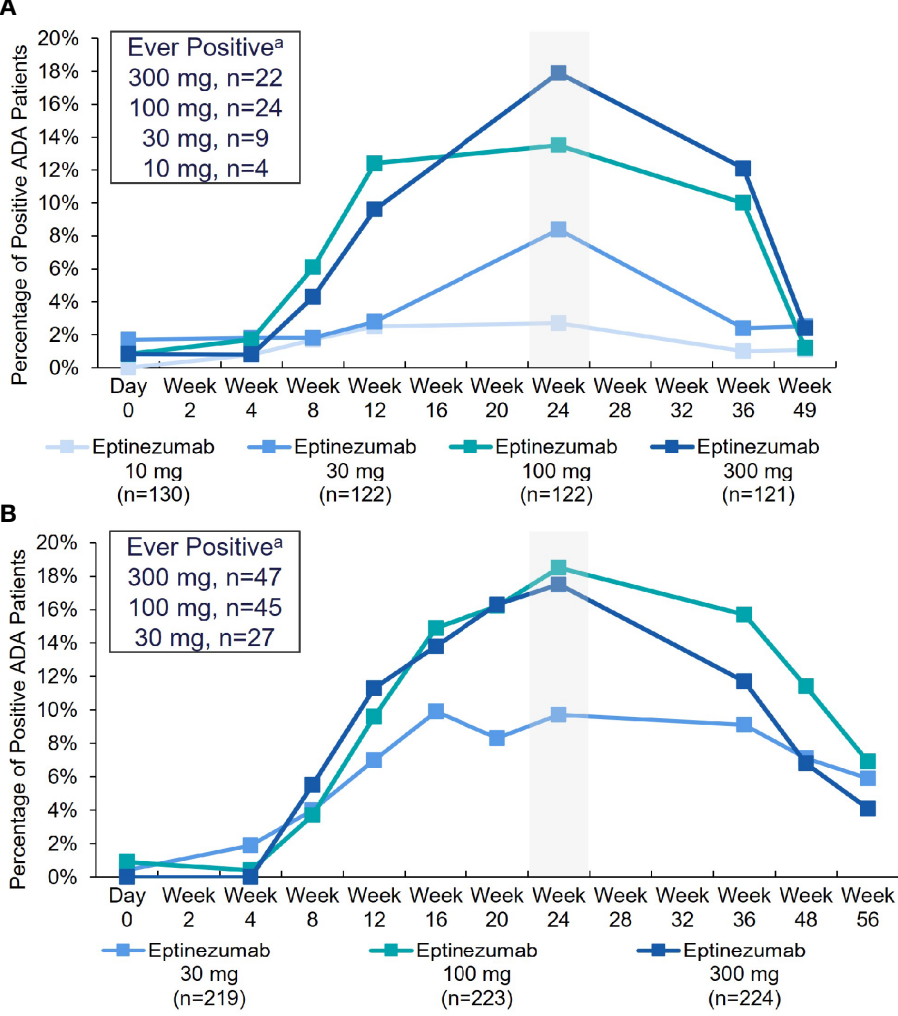

C
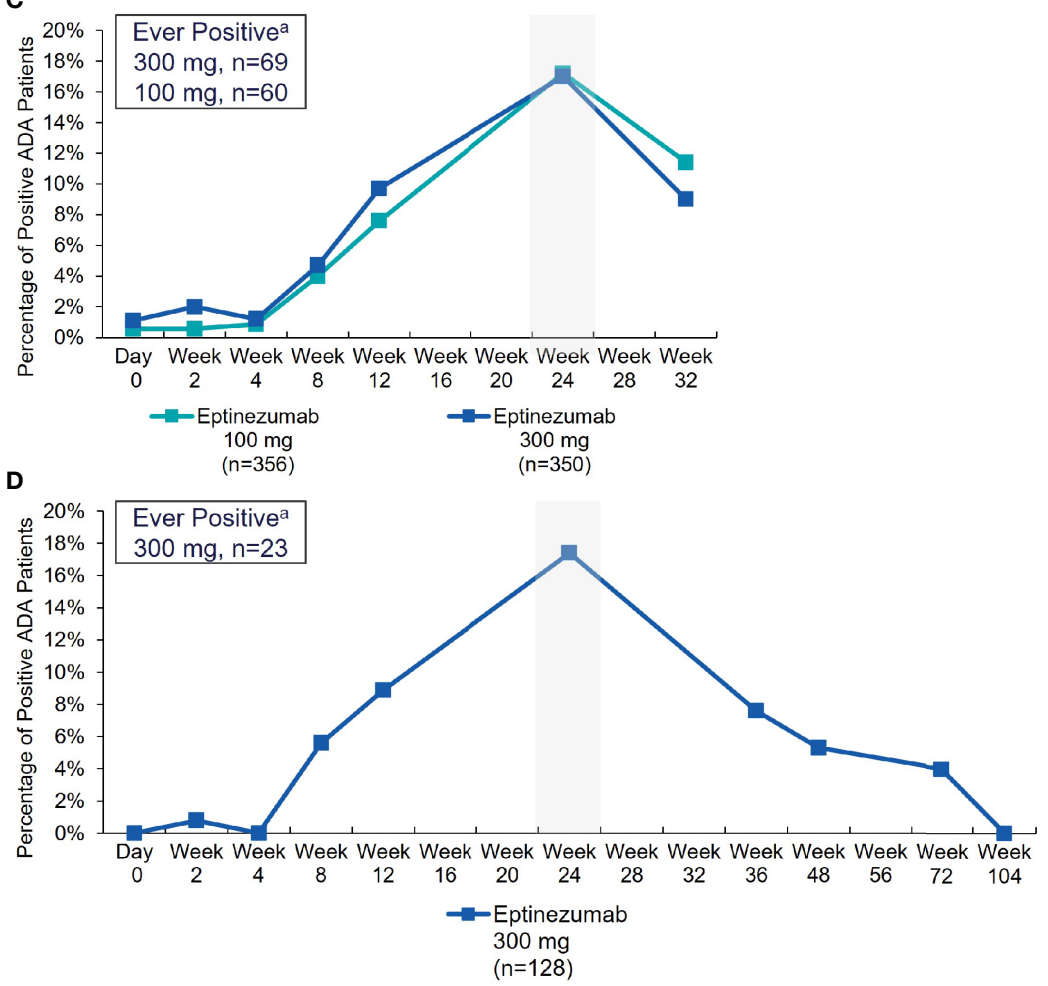

FIGURE 1 | Percentage of ADA-positive patients by visit and treatment (safety population) in: (A) Study 2, (B) PROMISE-1, (C) PROMISE-2, and (D) PREVAIL. ${ }^{a}$ Represents number of patients ever ADA positive over the entire time course analyzed for each study. Note: The denominator for the percentage is the number of patients with ADA sampling results per treatment group at each visit. ADA, anti-drug antibody. 
A

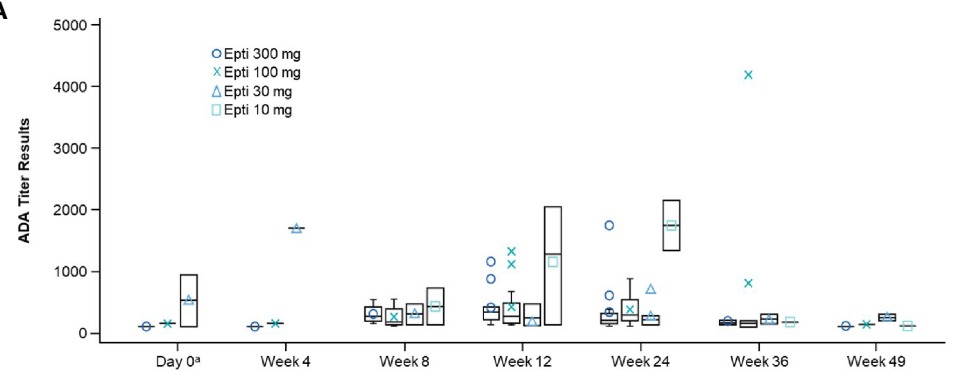

B

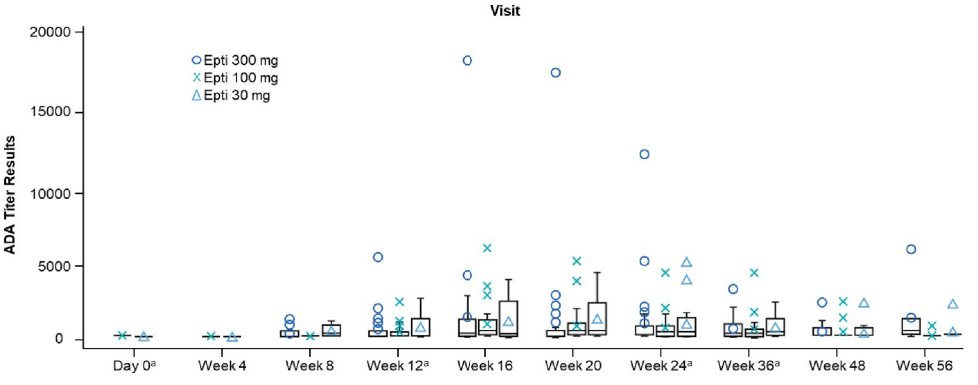

C
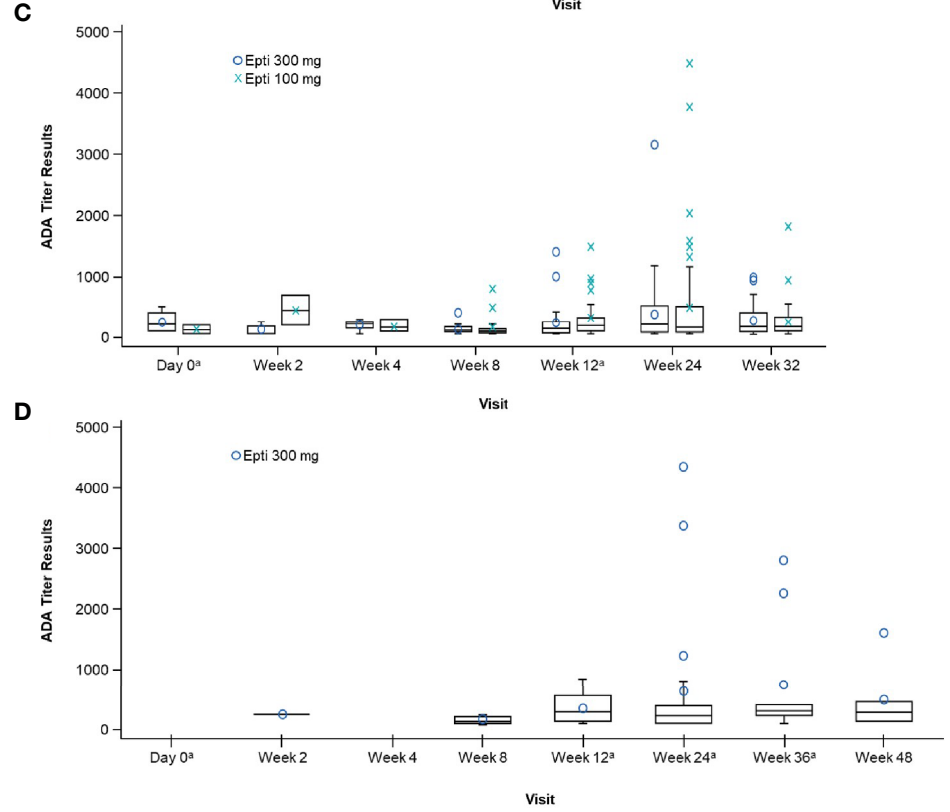

FIGURE 2 | Boxplot of positive ADA titer results by visit and treatment (safety population) in: (A) Study 2, (B) PROMISE-1, (C) PROMISE-2, and (D) PREVAIL. 'On visits that coincided with treatment, ADA was measured pre-dosing. Note: Only patients with a confirmed ADA-positive status are summarized at each visit. ADA, anti-drug antibody; Epti, eptinezumab. ADA titer refers to the value obtained within screening assay through a series of dilutions beginning with the minimal required dilution of confirmed positive samples.

The Pichia-expressed human IgG1 mAb containing the unrelated CDR sequence (ALD306) showed negligible inhibition of the signal. Tables $\mathbf{3}$ and $\mathbf{4}$ present the Specificity data from the PROMISE 1 and PROMISE 2 studies, respectively.

\section{Relationship to Drug Exposure}

Within each study, relationships between ADA or NAb status and mean eptinezumab plasma concentrations were evaluated at $\mathrm{C}_{\text {trough }}$, the most sensitive area of the exposure profile.
In both pivotal phase 3 studies (PROMISE-1 and PROMISE-2), mean $\mathrm{C}_{\text {trough }}$ concentrations were lower for the ADA- and NAbpositive subpopulations than for the ADA- and NAb-negative subpopulations at all evaluated doses and timepoints. At the week 24 visit, where the maximal incidence and titer of the ADA response was observed, mean $\mathrm{C}_{\text {trough }}$ plasma concentrations in the ADA-positive subgroups were 49.0 to $61.5 \%$ compared to that of the ADA-negative subgroups for eptinezumab $100 \mathrm{mg}$ and 67.0 to $81.1 \%$ compared to that of the ADA-negative subgroups for 
TABLE 3 | Specificity of ADA signals detected at all timepoints in PROMISE 1.

\begin{tabular}{|c|c|c|c|c|}
\hline \multirow[t]{2}{*}{ Statistic } & \multicolumn{3}{|c|}{ Eptinezumab dose } & \multirow[t]{2}{*}{ Overall $\mathrm{N}=666$} \\
\hline & 300 mg N=224 & $100 \mathrm{mg} \mathrm{N}=223$ & $30 \mathrm{mg} \mathrm{N}=219$ & \\
\hline Number of confirmed ADA-positive results for all timepoints & 176 & 188 & 118 & 482 \\
\hline Reactive with CHO ALD403 only, n (\%) & $130(73.9)$ & $137(72.9)$ & $84(71.2)$ & $351(72.8)$ \\
\hline Reactive with ALD306 only, n (\%) & $5(2.8)$ & $5(2.7)$ & $6(5.1)$ & $16(3.3)$ \\
\hline Reactive with CHO ALD403 + ALD306, n (\%) & $22(12.5)$ & $25(13.3)$ & $11(9.3)$ & $58(12.0)$ \\
\hline Not reactive with either CHO ALD403 or ALD306, n (\%) & $19(10.8)$ & $21(11.2)$ & $17(14.4)$ & $57(11.8)$ \\
\hline
\end{tabular}

$A D A$, anti-drug antibody; $\mathrm{CHO}$, Chinese hamster ovary expressed antibody.

TABLE 4 | Specificity of ADA signals detected in study eptinezumab PROMISE 2 Study (all timepoints).

\begin{tabular}{|c|c|c|c|}
\hline \multirow[t]{2}{*}{ Statistic } & \multicolumn{2}{|c|}{ Eptinezumab dose } & \multirow[t]{2}{*}{ Overall $\mathrm{N}=706$} \\
\hline & $300 \mathrm{mg} \mathrm{N}=350$ & $100 \mathrm{mg} \mathrm{N}=356$ & \\
\hline Number of confirmed ADA-positive results for all timepoints & 145 & 138 & 283 \\
\hline Reactive with $\mathrm{CHO}$ Eptinezumab only, n (\%) & $100(69.0)$ & 97 (70.3) & $197(69.6)$ \\
\hline Reactive with ALD306 only, n (\%) & $14(9.7)$ & $10(7.2)$ & $24(8.5)$ \\
\hline Reactive with $\mathrm{CHO}$ Eptinezumab + ALD306, n (\%) & $10(6.9)$ & $23(16.7)$ & $33(11.7)$ \\
\hline Not reactive with either CHO Eptinezumab or ALD306, n (\%) & $23(15.9)$ & $11(8.0)$ & $34(12.0)$ \\
\hline
\end{tabular}

$A D A$, anti-drug antibody; $\mathrm{CHO}$, Chinese hamster ovary cell expressed antibody.

eptinezumab $300 \mathrm{mg}$ in PROMISE-1 and PROMISE2 , respectively.

In spite of the decrease observed in the mean $\mathrm{C}_{\text {trough }}$ plasma concentrations in the ADA-positive subgroups in both studies, mean $\mathrm{C}_{\text {trough }}$ levels at week 24 following eptinezumab $100 \mathrm{mg}$ or $300 \mathrm{mg}$ remained above established plasma concentrations that support the $90 \%$ maximal efficacy (EC ${ }_{90}$ ) thresholds (18). In PROMISE-2, mean $\mathrm{C}_{\text {trough }}$ levels at week 24 were $\geq 6 \%$ above the established $\mathrm{EC}_{90}$ for CM patients $(0.98 \mu \mathrm{g} / \mathrm{mL})$, at $3.47 \mu \mathrm{g} / \mathrm{mL}$ and $10.18 \mu \mathrm{g} / \mathrm{mL}$ in the eptinezumab 100 - and 300-mg groups, respectively. Similar observations were found in the PROMISE-1 study, where the drug levels remained above the $\mathrm{EC}_{90}$ for $\mathrm{EM}$ patients $(1.65 \mu \mathrm{g} / \mathrm{mL})$, even among the highest ADA titers. These data support the observed absence of a negative influence on efficacy with a conclusion that ADA-positive status is not associated with a clinically meaningful change in the extent of drug exposure following the intravenous administration of eptinezumab 100 or 300 mg every 12 weeks.

\section{Relationship to Efficacy}

Despite the presence of ADA or NAb, the migraine preventive efficacy of eptinezumab was maintained. The change in MMDs by ADA status was remarkably consistent for ADA-positive vs ADAnegative patients in the three studies in which this relationship was evaluated (Study 2, PROMISE 1, and PROMISE 2; Figure 3). Similar results were obtained for the NAb-positive vs NAbnegative subpopulations (Table 5). Additionally, ADA titer had no apparent impact on efficacy in the 100- or 300-mg eptinezumab treatment groups, indicating that increased ADA titer did not result in a decrease in the eptinezumab efficacy.

The ANCOVA analysis of data from the full analysis population (i.e., all patients treated with eptinezumab, including 30,100 , and $300 \mathrm{mg}$ ) showed a highly comparable reduction in frequency of migraine days for the weeks 1-12 and weeks 13-24 treatment periods (Table 6), as well for the overall weeks 1-24 treatment period, for the ADA-positive vs ADA-negative subpopulations. There was no apparent relationship between efficacy (reduction in frequency of MMDs for weeks 1-24) with $\mathrm{ADA}$-positive status, $\mathrm{ADA}$ titer, or NAb-positive status for the $100-\mathrm{mg}$ or 300-mg treatment groups.

\section{Relationship to Safety}

Across the five studies, the incidences of TEAEs were in general similar in ADA ever-positive patients (ever-positive meaning a confirmed positive result at any single visit) and ADA-negative patients, and no clear differences were seen between the eptinezumab doses. Similarly, there were no clear differences of AESI incidences between ADA ever-positive patients and ADAnegative patients (Tables 7 and 8 ).

There were no cases of anaphylaxis or severe hypersensitivity reactions observed in study 1 , study 2 , PROMISE-1, or PROMISE2. There was 1 serious treatment-emergent adverse event of special interest of anaphylactic reaction of Grade 2 severity reported in PREVAIL, where the serious criteria of medically important was reported by the investigator. The Sponsor assessed the event using clinical diagnostic criteria as outlined by the second symposium on the definition and management of anaphylaxis (23) and determined the adverse event may have been better described by the preferred term (PT) of allergic reaction under the system organ class (SOC) of immune system disorders (9).

Within the safety populations for study 2, PROMISE-1, PROMISE-2, and PREVAIL, 24/1995 (1.2\%) eptinezumabtreated patients experienced mild or moderate adverse events that were coded to the PT of hypersensitivity. There was no apparent relationship to pre-existing or treatment-emergent ADA- or NAb-positive status or ADA titer category. 
A

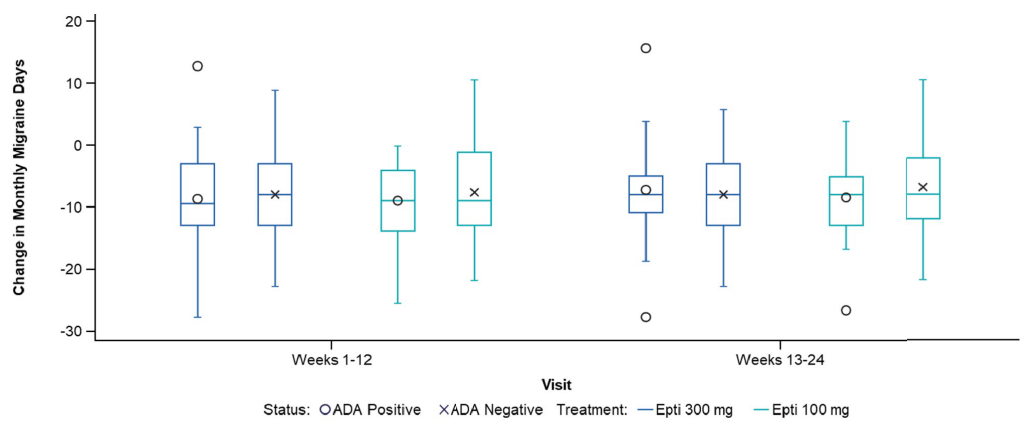

B

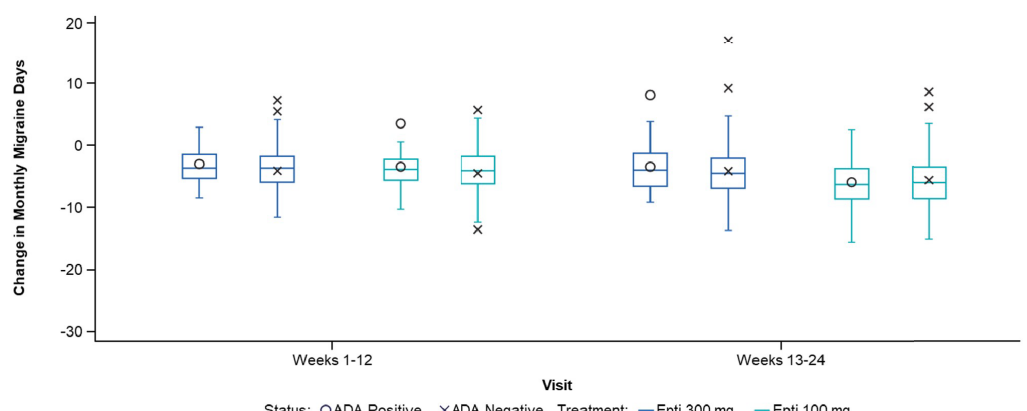

C

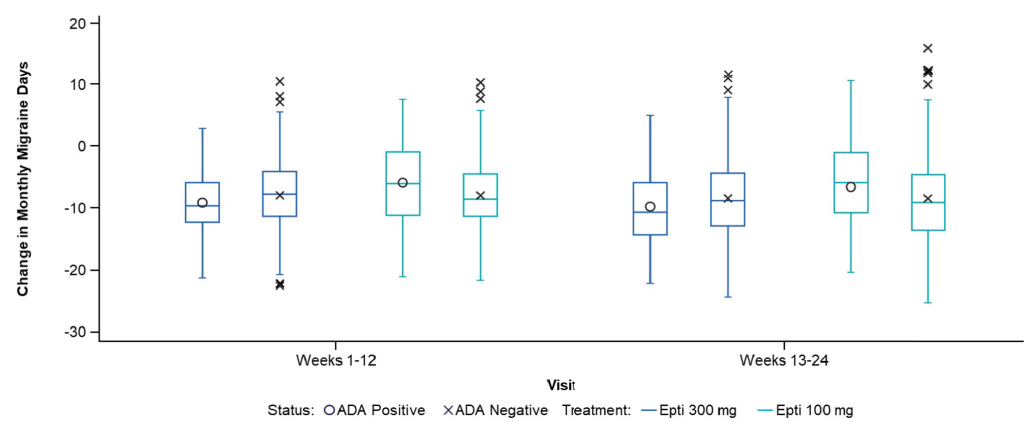

FIGURE 3 | Change in MMDs by ADA status in: (A) Study 2, (B) PROMISE-1, and (C) PROMISE-2. ADA, anti-drug antibody; Epti, eptinezumab, MMDs, monthly migraine days.

TABLE 5 | Change in monthly migraine days by ADA and NAb status in Study 2, PROMISE-1, and PROMISE-2 (full analysis population).

Study 2

Eptinezumab $300 \mathrm{mg}$

\section{ADA-positive}

Number of patients, $n$

Weeks 1-12 (SD)

Weeks 13-24 (SD)

Number of patients, $n$

Weeks 1-12 (SD)

Weeks 13-24 (SD)

Number of patients, $n$

Weeks 1-12 (SD)

Weeks 13-24 (SD)
A-positive ADA-negative

22

$-8.5(8.40)$

$-7.5(8.75)$

46

$-3.9(4.79)$

$-4.2(3.85)$

69

$-9.3(5.95)$

$-10.1(6.75)$
NAb-positive

91

$-8.1(6.71)$

$-8.3(6.51)$

176

$-4.4(3.56)$

$-5.0(4.02)$

281

$-8.1(5.95)$

$-8.7(6.70)$

$-9.7$

$-9.0(10.49)$

16

$-3.6(3.37)$

$-3.7(4.67)$

19

$-8.4(7.09)$

$-9.9(7.19)$
$-9.7(10.73)$

NAb-negative

$-6$.

$A D A$, anti-drug antibody; NAb, neutralizing antibodies.

\section{Eptinezumab 100 mg}

\section{ADA-positive ADA-negative}

23

$\begin{array}{cc}15 & 23 \\ -8.0(7.45) & -9.0(6.23)\end{array}$

$-6.8(8.13)$

PROMISE-1

30

$\begin{array}{cc}30 & 46 \\ -4.0(2.48) & -4.3(2.56) \\ -4.5(3.40) & -4.8(3.32)\end{array}$

PROMISE-2

95

$-7.3(7.01)$

$-6.8(6.89)$

8

$-8.8(4.74)$

$-7.8(6.11)$

22

175

$-3.8(3.38)$

$-4.6(3.56)$

$-4.1(2.48)$

$-4.7(3.51)$

$\begin{array}{cc}49 & 60 \\ -9.7(5.55) & -6.1(6.81)\end{array}$

$-10.1(6.72) \quad-6.6(6.89)$

NAb-positive

26

296

$-8.1(5.85)$

$-8.6(7.02)$

NAb-negative 
TABLE 6 | Change in monthly migraine days by ADA status in PROMISE-1 and PROMISE-2 (full analysis population; all dose levels combined)*.

\begin{tabular}{|c|c|c|c|c|}
\hline & \multicolumn{2}{|c|}{ PROMISE-1 } & \multicolumn{2}{|c|}{ PROMISE-2 } \\
\hline & ADA-positive $(n=119)$ & ADA-negative $(n=599)$ & ADA-positive $(n=129)$ & ADA-negative $(n=577)$ \\
\hline \multirow{3}{*}{$\begin{array}{l}\text { Weeks } 1-12, \text { mean change from baseline } \\
\text { Mean difference from ADA-negative }(95 \% \mathrm{Cl}) \\
\text { P-value }\end{array}$} & -4.4 & -4.3 & -7.7 & -8.0 \\
\hline & $-0.08(-0.70,0.54)$ & & $0.29(-0.83,1.42)$ & \\
\hline & 0.8022 & & 0.6081 & \\
\hline \multirow{3}{*}{$\begin{array}{l}\text { Weeks } 13-24, \text { mean change from baseline } \\
\text { Mean difference from ADA-negative }(95 \% \mathrm{Cl}) \\
\text { P-value }\end{array}$} & -4.9 & -4.9 & -8.4 & -8.6 \\
\hline & $0.08(-0.62,0.78)$ & & $0.27(-1.02,1.55)$ & \\
\hline & 0.8201 & & 0.6827 & \\
\hline \multirow{3}{*}{$\begin{array}{l}\text { Weeks } 1-24, \text { mean change from baseline } \\
\text { Mean difference from ADA-negative }(95 \% \mathrm{Cl}) \\
\text { P-value }\end{array}$} & -4.6 & -4.6 & -8.1 & -8.3 \\
\hline & $0.00(-0.62,0.62)$ & & $0.28(-0.87,1.43)$ & \\
\hline & 0.9972 & & 0.6306 & \\
\hline
\end{tabular}

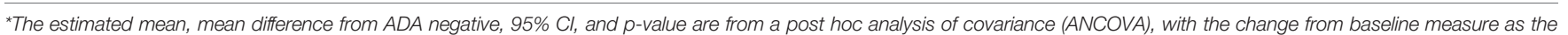

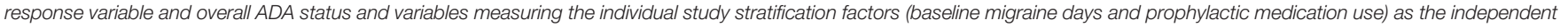
variables. ADA, anti-drug antibody; 95\% Cl, 95\% confidence interval.

TABLE 7 | Summary of TEAEs and AESIs by anti-eptinezumab antibody (ADA) status for PROMISE-1.

\begin{tabular}{|c|c|c|c|c|c|c|c|}
\hline \multirow[t]{2}{*}{ Treatment } & \multicolumn{2}{|c|}{ Eptinezumab 300 mg } & \multicolumn{2}{|c|}{ Eptinezumab 100 mg } & \multicolumn{2}{|c|}{ Eptinezumab 30 mg } & \multirow{2}{*}{$\begin{array}{c}\text { Placebo } \\
(n=222)\end{array}$} \\
\hline & $\begin{array}{l}\text { Ever positive } \\
\qquad(n=47)\end{array}$ & $\begin{array}{c}\text { Negative } \\
(\mathrm{n}=177)\end{array}$ & $\begin{array}{l}\text { Ever positive } \\
\qquad(n=45)\end{array}$ & $\begin{array}{c}\text { Negative } \\
(n=178)\end{array}$ & $\begin{array}{l}\text { Ever positive } \\
\qquad(n=27)\end{array}$ & $\begin{array}{c}\text { Negative } \\
(n=192)\end{array}$ & \\
\hline $\begin{array}{l}\text { Number of patients with any } \\
\text { TEAE (\%) }\end{array}$ & $31(66.0 \%)$ & $98(55.4 \%)$ & 26 (57.8\%) & 115 (64.6) & $16(59.3 \%)$ & 112 (58.3\%) & 132 (59.5\%) \\
\hline $\begin{array}{l}\text { Number of patients with any } \\
\text { AESI (\%) }\end{array}$ & 8 (17.0\%) & 14 (7.9\%) & 5 (11.1\%) & 18 (10.1\%) & 0 & 22 (11.5\%) & 11 (5.0\%) \\
\hline
\end{tabular}

AESI, adverse event of special interest; TEAE, treatment-emergent adverse event. Ever positive represents a confirmed positive result at any single visit.

TABLE 8 | Summary of TEAEs and AESI by anti-eptinezumab antibody (ADA) status for PROMISE-2.

\begin{tabular}{|c|c|c|c|c|c|}
\hline \multirow[t]{2}{*}{ Treatment group } & \multicolumn{2}{|c|}{ Eptinezumab $300 \mathrm{mg}$} & \multicolumn{2}{|c|}{ Eptinezumab $100 \mathrm{mg}$} & \multirow[t]{2}{*}{ Placebo $(n=366$} \\
\hline & Ever positive $(n=69)$ & Negative $(n=281)$ & Ever positive $(n=60)$ & Negative $(n=296)$ & \\
\hline Number of patients with any TEAE, $n(\%)$ & $33(47.8 \%)$ & $149(53.0 \%)$ & $31(51.7 \%)$ & $124(41.9 \%)$ & $171(46.7 \%)$ \\
\hline Number of patients with any AESI, $n(\%)$ & $5(7.2 \%)$ & $30(10.7 \%)$ & $7(11.7 \%)$ & $16(5.4 \%)$ & $18(4.9 \%)$ \\
\hline
\end{tabular}

AESI, adverse event of special interest; TEAE, treatment-emergent adverse event. Ever positive represents a confirmed positive result at any single visit.

\section{DISCUSSION}

In this integrated analysis of data from five clinical studies of eptinezumab for the preventive treatment of migraine, the overall incidences of treatment-emergent $\mathrm{ADA}(15.9 \%)$ and $\mathrm{NAb}(6.2 \%)$ were slightly higher than other anti-CGRP mAbs indicated as migraine preventives (24); however, the presence of $\mathrm{ADA}$ and NAb did not appear to influence treatment efficacy or safety. Efficacy for migraine prevention was comparable in ADApositive and ADA-negative subpopulations. There were no cases of severe hypersensitivity reactions and only 1 serious AESI of anaphylactic reaction, which did not fulfill the clinical diagnostic criteria for anaphylactic reactions as outlined by the second symposium on the definition and management of anaphylaxis (23). There was no evidence for a risk of immune complexrelated hypersensitivity, consistent with the relatively low ADA titers observed for all eptinezumab dose levels, and there was no relationship between the $\mathrm{ADA}$ or $\mathrm{NAb}$ status and the incidence or severity of other adverse events of special interest. As the incidence of treatment-emergent ADAs in these studies consistently peaked at week 24 , it is likely that any potential negative effects of immunogenicity would have manifested by this timepoint. Thus, these findings reduce uncertainty about the possible impact of immunogenicity on the sustainability of the treatment response and the potential for treatment-related hypersensitivity reactions with long-term administration.

Overall, the ADA frequency and titer profiles indicate the maximum amplitude of the treatment-emergent ADA response is attained following the second of the 3-monthly dose administrations regardless of the eptinezumab dose level. After 24 weeks the ADA titer declined, consistent with the half-life of endogenous human IgG (21-25 days). The reason for this decline is likely due to a combination of moderate to low titer levels, minimal boosting, and a natural clearance mechanism. ADA would be expected to be eliminated after approximately 5 halflives, which amounts to about 16 weeks. Based on these data, the dynamics (in terms of both frequency and magnitude) were highly comparable for the 100- and 300-mg eptinezumab treatment 
groups through the complete time-course for each clinical study. Characterization of the ADA responses showed reactivity was directed toward the primary amino acid sequence (CDRs) of eptinezumab, which is typical for most therapeutic mAbs (13).

Although the analysis of potential relationships between $\mathrm{ADA} / \mathrm{NAb}$ and drug exposure revealed differences in $\mathrm{C}_{\text {trough }}$ plasma concentrations between ADA-positive and ADAnegative patients, it did not identify any associated clinical impact. Though a decrease in serum concentration was observed, the drug levels remained above $\mathrm{EC}_{90}$, even among the highest ADA titers, supporting the conclusion that ADApositive status is not associated with a clinically meaningful change in the extent of drug exposure.

The molecular design of eptinezumab effectively minimized product-related factors known to be associated with increased immunogenicity among mAbs. As demonstrated in the specificity evaluation of $\mathrm{ADA}$, the responses were mainly reactive with the CHO-expressed ALD403, which has the same CDRs as ALD403 but is produced in mammalian cells rather than yeast cells, resulting in differences in the IgG1 framework region, IgG1 Fc region, or Pichia-derived glycans. Using the CHO ALD403 antibody with the same amino acid sequence as eptinezumab, expressed from a different host expression system, enabled pinpointing of the response directly to the CDRs of eptinezumab. Thus treatment-emergent ADAs associated with eptinezumab administration are not likely to enhance responses toward subsequent treatment with other therapeutic mAb products.

The clinical studies included in this analysis were designed to capture the dynamics of a potential humoral immune response to eptinezumab treatment, and descriptive analyses were applied to interpret the relationship of $\mathrm{ADA} / \mathrm{NAb}$ signals to drug exposure, efficacy, and safety. Limitations of this analysis include the clinical trial population, which does not reflect all patients with episodic or chronic migraine, and long-term effects, which were only reported up to 1 year here. Further studies in a broader population with migraine and longer-term studies could further elucidate the efficacy and safety of eptinezumab (25).

In conclusion, the results of this analysis suggest that the clinical benefits and safety of eptinezumab for the preventive treatment of migraine are not impacted by immunogenicity.

\section{DATA AVAILABILITY STATEMENT}

In accordance with EFPIA's and PhRMA's "Principles for Responsible Clinical Trial Data Sharing” guidelines, Lundbeck is committed to responsible sharing of clinical trial data in a manner that is consistent with safeguarding the privacy of patients,

\section{REFERENCES}

1. VYEPTI [package insert]. Bothell, WA: Lundbeck Seattle BioPharmaceuticals, Inc; (2021).

2. Baker B, Schaeffler B, Cady R, Latham J, Whitaker T, Smith J. Rational Design of a Monoclonal Antibody (mAB) Inhibiting Calcitonin Gene-Related Peptide (CGRP), ALD403, Intended for the Prevention of Migraine (P2.155). Neurology (2017) 88. respecting the integrity of national regulatory systems, and protecting the intellectual property of the sponsor. The protection of intellectual property ensures continued research and innovation in the pharmaceutical industry. Deidentified data are available to those whose request has been reviewed and approved through an application submitted to https:/www.lundbeck.com/global/ourscience/clinical-data-sharing.

\section{ETHICS STATEMENT}

Approval for each study was provided by the independent ethics committee or institutional review board of the study sites. All studies were conducted in accordance with Good Clinical Practice guidelines, the principles of the Declaration of Helsinki, and all applicable regulatory requirements. Patients provided written informed consent prior to initiation of any study procedures.

\section{AUTHOR CONTRIBUTIONS}

$\mathrm{SP}, \mathrm{DB}, \mathrm{BA}, \mathrm{RC}, \mathrm{BS}, \mathrm{BB}$, and JL contributed to conception and design of this work. SP and $\mathrm{DB}$ contributed to the acquisition, analysis, and interpretation of this work. SP, DB, BA, RC, BS, BB, and JL contributed to the drafting and revision of this work. SP, $\mathrm{DB}, \mathrm{BA}, \mathrm{RC}, \mathrm{BS}, \mathrm{BB}$, and JL provided final approval of the version to be published. All authors agree to be accountable for the content of the work.

\section{FUNDING}

This study was funded by H. Lundbeck A/S (Copenhagen, Denmark).

\section{ACKNOWLEDGMENTS}

The authors thank Mary Tom, PharmD, and Sarah Russell, PhD, of The Medicine Group (New Hope, PA, USA), for providing medical writing support, which was funded by $\mathrm{H}$. Lundbeck A/S (Copenhagen, Denmark) in accordance with Good Publication Practice guidelines.

\section{SUPPLEMENTARY MATERIAL}

The Supplementary Material for this article can be found online at: https://www.frontiersin.org/articles/10.3389/fimmu.2021. 765822/full\#supplementary-material

3. Dodick DW, Goadsby PJ, Silberstein SD, Lipton RB, Olesen J, Ashina M, et al. Safety and Efficacy of ALD403, an Antibody to Calcitonin Gene-Related Peptide, for the Prevention of Frequent Episodic Migraine: A Randomised, Double-Blind, Placebo-Controlled, Exploratory Phase 2 Trial. Lancet Neurol (2014) 13:1100-7. doi: 10.1016/S1474-4422(14)70209-1

4. Dodick DW, Lipton RB, Silberstein S, Goadsby PJ, Biondi D, Hirman J, et al. Eptinezumab for Prevention of Chronic Migraine: A Randomized Phase 2b Clinical Trial. Cephalalgia (2019) 39:1075-85. doi: 10.1177/0333102419858355 
5. Ashina M, Saper J, Cady R, Schaeffler B, Biondi DM, Hirman J, et al. Eptinezumab in Episodic Migraine: A Randomized, Double-Blind, PlaceboControlled Study (PROMISE-1). Cephalalgia (2020) 40:241-54. doi: 10.1177/ 0333102420905132

6. Smith TR, Janelidze M, Chakhava G, Cady R, Hirman J, Allan B, et al. Eptinezumab for the Prevention of Episodic Migraine: Sustained Effect Through 1 Year of Treatment in the PROMISE-1 Study. Clin Ther (2020) 42:2254-65.e3. doi: 10.1016/j.clinthera.2020.11.007

7. Lipton RB, Goadsby PJ, Smith J, Schaeffler BA, Biondi DM, Hirman J, et al. Efficacy and Safety of Eptinezumab in Patients With Chronic Migraine. PROMISE-2. Neurology (2020) 94:e1365-77. doi: 10.1212/WNL. 0000000000009169

8. Silberstein S, Diamond M, Hindiyeh NA, Biondi DM, Cady R, Hirman J, et al. Eptinezumab for the Prevention of Chronic Migraine: Efficacy and Safety Through 24 Weeks of Treatment in the Phase 3 PROMISE-2 (Prevention of Migraine via Intravenous ALD403 Safety and Efficacy-2) Study. J Headache Pain (2020) 21:120. doi: 10.1186/s10194-020-01186-3

9. Kudrow D, Cady RK, Allan B, Pederson SM, Hirman J, Mehta LR, et al. LongTerm Safety and Tolerability of Eptinezumab in Patients With Chronic Migraine: A 2-Year, Open-Label, Phase 3 Trial. BMC Neurol (2021) 21:126. doi: 10.1186/s12883-021-02123-w

10. Smith TR, Spierings ELH, Cady R, Hirman J, Schaeffler B, Shen V, et al. Safety and Tolerability of Eptinezumab in Patients With Migraine: A Pooled Analysis of 5 Clinical Trials. J Headache Pain (2021) 22:16. doi: 10.1186/ s10194-021-01227-5

11. Yan Z, Xue T, Chen S, Wu X, Yang X, Liu G, et al. Different Dosage Regimens of Eptinezumab for the Treatment of Migraine: A Meta-Analysis From Randomized Controlled Trials. J Headache Pain (2021) 22:10. doi: 10.1186/ s10194-021-01220-y

12. Rosenberg AS. Immunogenicity of Biological Therapeutics: A Hierarchy of Concerns. Dev Biol (Basel) (2003) 112:15-21.

13. Harding FA, Stickler MM, Razo J, Dubridge RB. The Immunogenicity of Humanized and Fully Human Antibodies: Residual Immunogenicity Resides in the CDR Regions. MAbs (2010) 2:256-65. doi: 10.4161/mabs.2.3.11641

14. Shankar G, Arkin S, Cocea L, Devanarayan V, Kirshner S, Kromminga A, et al. Assessment and Reporting of the Clinical Immunogenicity of Therapeutic Proteins and Peptides-Harmonized Terminology and Tactical Recommendations. AAPS J (2014) 16:658-73. doi: 10.1208/s12248-014-9599-2

15. FDA. Guidance for Industry: Immunogenicity Assessment for Therapeutic Protein Products (2014). Available at: https://www.fda.gov/media/85017/ download (Accessed February 2, 2021).

16. FDA. Guidance for Industry: Assay Development and Validation for Immunogenicity Testing of Therapeutic Protein Products (2016). Available at: https://www.fda.gov/media/77796/download (Accessed February 2, 2021).

17. Smith TR, Janelidze M, Chakhava G, Cady R, Hirman J, Allan B, et al. Eptinezumab for the Prevention of Episodic Migraine: Sustained Effect Through 1 Year of Treatment in the PROMISE-1 Study. Clin Ther (2020) 42:2254-65.e3. doi: 10.1016/j.clinthera.2020.11.007

18. Baker B, Schaeffler B, Beliveau M, Rubets I, Pederson S, Trinh M, et al. Population Pharmacokinetic and Exposure-Response Analysis of Eptinezumab in the Treatment of Episodic and Chronic Migraine. Pharmacol Res Perspect (2020) 8:e00567. doi: 10.1002/prp2.567
19. FDA. Guidance for Industry: Assay Development for Immunogenicity Testing of Therapeutic Proteins (2009) (Accessed February 2, 2021).

20. FDA. Bioanalytical Method Validation: Guidance for Industry (2018). Available at: https://www.fda.gov/files/drugs/published/BioanalyticalMethod-Validation-Guidance-for-Industry.pdf (Accessed February 2, 2021).

21. USP Chapter 1106. United States Pharmacopeia. Chapter 1106. Immunogenicity Assays - Design and Validation of Immunoassays to Detect Anti-Drug Antibodies (2012). Available at: http://www.usp.org (Accessed February 2, 2021).

22. EMEA. 2017 Guideline on Immunogenicity Assessment of Therapeutic Proteins (EMEA/CHMP/BMWP/14327/2006 Rev 1), 18 May (2017). Available at: http://www.ema.europa.eu/docs/en_GB/document_library/Scientific_ guideline/2017/06/WC500228861.pdf (Accessed February 2, 2021).

23. Sampson HA, Munoz-Furlong A, Campbell RL, Adkinson NF Jr, Bock SA, Branum A, et al. Second Symposium on the Definition and Management of Anaphylaxis: Summary Report-Second National Institute of Allergy and Infectious Disease/Food Allergy and Anaphylaxis Network Symposium. J Allergy Clin Immunol (2006) 117:391-7. doi: 10.1016/ j.jaci.2005.12.1303

24. Cohen JM, Ning X, Kessler Y, Rasamoelisolo M, Campos VR, Seminerio MJ, et al. Immunogenicity of Biologic Therapies for Migraine: A Review of Current Evidence. J Headache Pain (2021) 22:3. doi: 10.1186/s10194-02001211-5

25. Spuntarelli V, Negro A, Luciani M, Bentivegna E, Martelletti P. Eptinezumab for the Treatment of Migraine. Expert Opin Biol Ther (2021) 21:999-1011. doi: $10.1080 / 14712598.2021 .1931678$

Conflict of Interest: All authors were full-time employees of company $\mathrm{H}$. Lundbeck A/S (formally known as Alder BioPharmaceuticals), or one of its subsidiary companies, at the time of study and during manuscript preparation. BS has patents: Eptinezumab in the acute treatment of Migraines pending, and Eptinezumab in the treatment of $\mathrm{MOH}$ pending. SP, BB, and RC are full-time employees of $\mathrm{H}$. Lundbeck A/S or one of its subsidiary companies.

The authors declare that this study received funding from $H$. Lundbeck A/S. The funder had the following involvement with the study: design and conduct of the study; data collection, management, and analysis; and preparation, review, and approval of the manuscript for publication.

Publisher's Note: All claims expressed in this article are solely those of the authors and do not necessarily represent those of their affiliated organizations, or those of the publisher, the editors and the reviewers. Any product that may be evaluated in this article, or claim that may be made by its manufacturer, is not guaranteed or endorsed by the publisher.

Copyright (c) 2021 Pederson, Biondi, Allan, Cady, Schaefler, Baker and Latham. This is an open-access article distributed under the terms of the Creative Commons Attribution License (CC BY). The use, distribution or reproduction in other forums is permitted, provided the original author(s) and the copyright owner(s) are credited and that the original publication in this journal is cited, in accordance with accepted academic practice. No use, distribution or reproduction is permitted which does not comply with these terms. 